This item was submitted to Loughborough's Research Repository by the author.

Items in Figshare are protected by copyright, with all rights reserved, unless otherwise indicated.

\title{
Numerical modelling of micro-machining of f.c.c. single crystal: influence of strain gradients
}

PLEASE CITE THE PUBLISHED VERSION

http://dx.doi.org/10.1016/j.commatsci.2014.05.058

PUBLISHER

(C) Elsevier

VERSION

AM (Accepted Manuscript)

\section{PUBLISHER STATEMENT}

This work is made available according to the conditions of the Creative Commons Attribution-NonCommercialNoDerivatives 4.0 International (CC BY-NC-ND 4.0) licence. Full details of this licence are available at: https://creativecommons.org/licenses/by-nc-nd/4.0/

\section{LICENCE}

CC BY-NC-ND 4.0

\section{REPOSITORY RECORD}

Demiral, Murat, Anish Roy, Tamer El Sayed, and Vadim V. Silberschmidt. 2019. "Numerical Modelling of Micro-machining of F.c.c. Single Crystal: Influence of Strain Gradients". figshare. https://hdl.handle.net/2134/25641. 


\title{
Numerical modelling of micro-machining of f.c.c. single crystal: Influence of strain gradients
}

\author{
Murat Demiral ${ }^{\mathrm{a}}$, Anish Roy ${ }^{\mathrm{b}, *}$, Tamer El Sayed ${ }^{\mathrm{a}}$, Vadim V. Silberschmidt ${ }^{\mathrm{b}}$ \\ ${ }^{a}$ Computational Solid Mechanics Laboratory, Division of Physical Sciences and Engineering, King Abdullah University of Science and Technology (KAUST), Saudi Arabia \\ ${ }^{\mathrm{b}}$ Wolfson School of Mechanical and Manufacturing Engineering, Loughborough University, LE11 3TU, UK
}

\author{
A B S T R A C T
}

\begin{abstract}
A micro-machining process becomes increasingly important with the continuous miniaturization of components used in various fields from military to civilian applications. To characterise underlying micromechanics, a 3D finite-element model of orthogonal micro-machining of f.c.c. single crystal copper was developed. The model was implemented in a commercial software ABAQUS/Explicit employing a userdefined subroutine VUMAT. Strain-gradient crystal-plasticity and conventional crystal-plasticity theories were used to demonstrate the influence of pre-existing and evolved strain gradients on the cutting process for different combinations of crystal orientations and cutting directions.
\end{abstract}

\section{Introduction}

Owing to continuous miniaturization, many modern high-technology applications such as medical and optical devices, thermal barrier coatings, electronics, micro- and nano-electro-mechanical systems increasingly use components with sizes of a few microns or smaller [1]. To manufacture such components, often with complex geometries, mechanical micro-machining is widely used [2-5]. A machining process such as cutting at the micro-scale differs significantly from its macro-scale counterpart in terms of cutting forces, chip formation and surface morphology [6,7]. Due to the very nature of the micro-machining process, its process zone is limited to a single or a few grains of machined material. Since single crystals are known to be highly anisotropic in their physical properties, it is not surprising that several experimental studies demonstrated dependence of cutting behaviour on crystallographic orientation, slip system and dislocation activity [8].

Compared to experimental studies, a limited number of modelling studies for single-grain micromachining is reported in the literature. Among the analytical models, Sato et al. [9] used a Schmid factor to obtain active slip systems during machining, Lee and Zhou [10] used an effective Taylor factor to predict the effective shear angle in single-crystal cutting and studies in $[11,12]$ considered minimization of the total power in the process zone to determine the shear angle and specific cutting energy.

\footnotetext{
* Corresponding author. Tel.: +44 1509227566.

E-mail address: A.Roy3@lboro.ac.uk (A. Roy).
}

Pen et al. [13] and Komanduri et al. [14] used quasi-continuum and molecular-dynamics simulation methods, respectively, to study the effect of crystal orientation and cutting direction on the deformation mechanism in nanometric cutting. Zahedi et al. [15] used smoothed particle hydrodynamics to model singlegrain cutting process. Recently, Tajalli et al. [16] analysed orthogonal micro-cutting of f.c.c. materials based on conventional crystal plasticity.

It is well known that the presence of strain gradients associated with non-uniform plastic deformation in small volumes becomes prominent at the micron or sub-micron scales $[17,18]$, which is often used to explain the observed size effect phenomena in bending [19], indentation [20] and other macroscopically inhomogeneous deformation regimes. In this context, it becomes imperative to account for strain gradients to accurately predict micro-scale material removal in machining models. To this end, this paper presents a 3D non-local elastic-viscoplastic crystal-plasticity finite-element (FE) model for micro-machining of f.c.c. single-crystal copper. To the authors' knowledge, this is the first study in the literature demonstrating the influence of strain gradients on the deformation response of crystalline materials in the machining process.

This paper is organized as follows: the theory for a strain-gradient crystal-plasticity theory with governing equations and details of the developed finite-element model of micro-cutting are introduced in Section 2. Section 3 demonstrates its predictive capabilities for different parameters such as crystallographic orientations, cutting directions and dimensions of a workpiece material including 
its validation against experimental data. The paper ends with concluding remarks in Section 4.

\section{Simulation procedure}

\subsection{EMSGCP constitutive model}

An enhanced modelling scheme for a strain-gradient crystalplasticity (EMSGCP) theory proposed by Demiral [21] was used in the simulations. The essential equations of EMSGCP are summarised below. In the following, a bold symbol denotes a vector or a tensor and a dot superposed on a symbol indicates a material time derivative. The operator $\times$ is a vector product, a gradient operator is indicated by $\nabla$, incremental changes in variables due to temporal evolution are indicated by a $\Delta$ symbol. A subscript $G$ indicates geometrically necessary dislocations (GNDs) and $S$ implies statistically stored dislocations (SSDs).

In the EMSGCP theory, the initial strength of a slip systems $\left(\left.g_{T}^{\alpha}\right|_{t=0}\right)$, i.e. the critical resolved shear stress (CRSS), is governed by pre-existing GNDs in the workpiece together with SSDs, i.e. $\left.g_{T}^{\alpha}\right|_{t=0}=\left.g_{S}^{\alpha}\right|_{t=0}+\left.g_{G}^{\alpha}\right|_{t=0}$. In this theory, $\left.g_{S}^{\alpha}\right|_{t=0}$ and $\left.g_{G}^{\alpha}\right|_{t=0}$ were linked with initial $\operatorname{SSD}\left(\left.\rho_{S}^{\alpha}\right|_{t=0}\right)$ and GND $\left(\left.\rho_{G}^{\alpha}\right|_{t=0}\right)$ densities as $\left.g_{S}^{\alpha}\right|_{t=0}=K \sqrt{\left.\rho_{S}^{\alpha}\right|_{t=0}},\left.g_{G}^{\alpha}\right|_{t=0}=K \sqrt{\left.\rho\right|_{t=0}(\bar{S} / \bar{V})^{2}}$ via the constant, $K$, similar to the Taylor relation. The GND density term was expressed as a function of the normalized surface-to-volume $(\bar{S} / \bar{V})$ ratio (hence, dimensionless) for the component under study [22]. In the study the surface-to-volume ratio of the workpiece materials was normalized with an idealised workpiece geometry corresponding to $S / V=1 \mu \mathrm{m}^{-1}$.

The evolution of slip resistance during loading is the result of hardening due to the SSDs $\left(\Delta g_{S}^{\alpha}\right)$ and GNDs $\left(\Delta g_{G}^{\alpha}\right)$ on the slip system, which follows,

$g_{T}^{\alpha}=\left.g_{S}^{\alpha}\right|_{t=0}+\left.g_{G}^{\alpha}\right|_{t=0}+\sqrt{\left(\Delta g_{S}^{\alpha}\right)^{2}+\left(\Delta g_{G}^{\alpha}\right)^{2}}$

where

$\Delta g_{S}^{\alpha}=\sum_{\beta=1}^{N} h_{\alpha \beta} \Delta \gamma^{\beta}, \quad \Delta g_{G}^{\alpha}=\alpha_{T} \mu_{s} \sqrt{b n_{G}^{\alpha}}$.

Here $h_{\alpha \beta}, \alpha_{T}, \mu_{s}, b$ and $n_{G}^{\alpha}$ corresponds to the slip-hardening modulus, the Taylor coefficient, the shear modulus, the Burgers vector and the effective density of geometrically necessary dislocations, respectively.

The hardening model proposed by Peirce et al. [23] is used to represent $h_{\alpha \beta}$, as follows:

$h_{\alpha \alpha}=h_{0} \sec h^{2}\left|\frac{h_{0} \tilde{\gamma}}{\left.g_{T}^{\alpha}\right|_{\mathrm{sat}}-\left.g_{T}^{\alpha}\right|_{t=0}}\right|, \quad h_{\alpha \beta}=q h_{\alpha \alpha}(\alpha \neq \beta), \tilde{\gamma}=\sum_{\alpha} \int_{0}^{t}\left|\dot{\gamma}^{\alpha}\right| d t$,

where $h_{0}$ is the initial hardening parameter, $\left.g_{T}^{\alpha}\right|_{\text {sat }}$ is the saturation stress of the slip system $\alpha, q$ is the latent hardening ratio, and $\tilde{\gamma}$ is the Taylor cumulative shear strain on all slip systems. The effective GND density $\left(n_{G}^{\alpha}\right)$ is given by

$n_{G}^{\alpha}=\left|\mathbf{m}^{\alpha} \times \sum_{\beta} \mathbf{s}^{\alpha \beta} \nabla \gamma^{\beta} \times \mathbf{m}^{\beta}\right|$,

where $\mathbf{s}^{\alpha}$ is the slip direction, $\mathbf{m}^{\alpha}$ is the slip-plane normal, $\boldsymbol{S}^{\alpha \beta}=\boldsymbol{S}^{\alpha} . \boldsymbol{S}^{\beta}$ and $\nabla \gamma^{\beta}$ is the gradient of shear strain in each slip system. To calculate $\nabla \gamma^{\beta}$ the scheme proposed in Demiral et al. [22] is followed. The model was implemented in the implicit finite-element code ABAQUS/Explicit using the user-defined material subroutine (VUMAT) [24]. Relevant details can be found in [22].

In Eq. (2), $\dot{\gamma}^{\alpha}$ is the shearing rate on the slip system $\alpha$. A powerlaw representation was chosen for $\dot{\gamma}^{\alpha}$ : $\dot{\gamma}^{\alpha}=\dot{\gamma}_{0}^{\alpha} \operatorname{sgn}\left(\tau^{\alpha}\right)\left|\frac{\tau^{\alpha}}{g_{T}^{\alpha}}\right|^{n}$,

where $\dot{\gamma}_{0}^{\alpha}$ is the reference strain rate, $n$ is the macroscopic rate-sensitivity parameter, $\tau^{\alpha}$ is the resolved shear stress, $g_{T}^{\alpha}$ is the strength of the slip system $\alpha$ at the current time, and sgn ( $\&$ is the signum function of $\&$.

It should be noted that unlike the proposed EMSGCP theory the contribution of pre-existing GNDs in the workpiece is neglected in the mechanism-based strain-gradient crystal-plasticity (MBSGCP) theory proposed by Han et al. [25], i.e. the influence of pre-existing strain gradients is insignificant; hence, the CRSS value of slip systems depends only on SSDs. Consequently, in the classical CP theory the contribution from both incipient and evolving GNDs is not accounted for, i.e. the instantaneous strength of slip system is given by $g_{T}^{\alpha}=\left.g_{S}^{\alpha}\right|_{t=0}+\Delta g_{S}^{\alpha}$.

To demonstrate the relative contribution of the evolution of GNDs to the local hardening of the material in EMSGCP, an enhanced model of crystal-plasticity (EMCP) was introduced, where the incipient density of GNDs in the experimental samples is accounted for, but not GNDs evolving in the course of deformation. This model is recovered from the proposed EMSGCP theory by setting $\Delta g_{G}^{\alpha}=0$. A summary of the contribution from GNDs and SSDs from the various theories discussed here is given in Table 1.

Here, machining in a single crystal of copper, which has a f.c.c. crystalline structure, is studied. In such materials slip may occur on 12 individual slip systems represented by the family $\{111\}<110\rangle$.

\subsection{Finite-element implementation}

A FE model of orthogonal micro-machining cutting was developed. Dimensions of the workpiece sample used in the FE model were $20 \mu \mathrm{m} \times 20 \mu \mathrm{m} \times 0.48 \mu \mathrm{m}$ (Fig. 1 ). To discretise the sample 29600 eight-node linear brick elements (C3D8) were used (Fig. 2). A minimum element size of $60 \mathrm{~nm}$ was chosen in the process zone based on a compromise between accuracy and computational time. The cutting tool was modelled as a rigid body with a rake and clearance angles of $0^{\circ}$. The tool was displaced in the negative $x$-direction with velocity of $1300 \mathrm{~mm} / \mathrm{s}$ (Fig. 1). A depth of cut $\left(a_{p}\right)$ was set at $2 \mu \mathrm{m}$, and the maximum cutting length of $2.25 \mu \mathrm{m}$ was considered in the simulations. The bottom ( $x z$ plane, $y=0)$, left ( $y z$ plane, $x=0$ ) and part of the right face ( $y z$ plane, $x=20 \mu \mathrm{m}$ and $0 \leqslant y<18 \mu \mathrm{m}$ ) of the workpiece were constrained from displacing in all directions, while the front and back face ( $x y$ plane, $z=0$ and $z=-0.48 \mu \mathrm{m}$, respectively) was constrained from displacing in the $z$ direction only (Fig. 1). Friction was neglected throughout the simulation.

Chip separation from the workpiece material was achieved with the use of element-deletion module available in ABAQUS/Explicit. The magnitude of Taylor cumulative shear strain on all slip systems was considered as parameter for the failure criteria. Shear failure occurred in the damageable zone when $\tilde{\gamma}$ reached a critical value, similar to one in [26],

$\tilde{\gamma}=\sum_{\alpha} \int_{0}^{t}\left|\dot{\gamma}^{\alpha}\right| d t=3$

Table 1

Summary of different strain-gradient and conventional CP theories.

\begin{tabular}{|c|c|c|c|c|}
\hline & $\left.g_{S}^{\alpha}\right|_{t=0}$ & $\left.g_{G}^{\alpha}\right|_{t=0}$ & $\Delta g_{S}^{\alpha}$ & $\Delta g_{G}^{\alpha}$ \\
\hline EMSGCP & $\nu$ & $\nu$ & $\nu$ & $\nu$ \\
\hline EMCP & $\nu$ & $v$ & $v$ & - \\
\hline MBSGCP & $\nu$ & - & 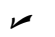 & v \\
\hline $\mathrm{CP}$ & $\nu$ & - & $\nu$ & - \\
\hline
\end{tabular}




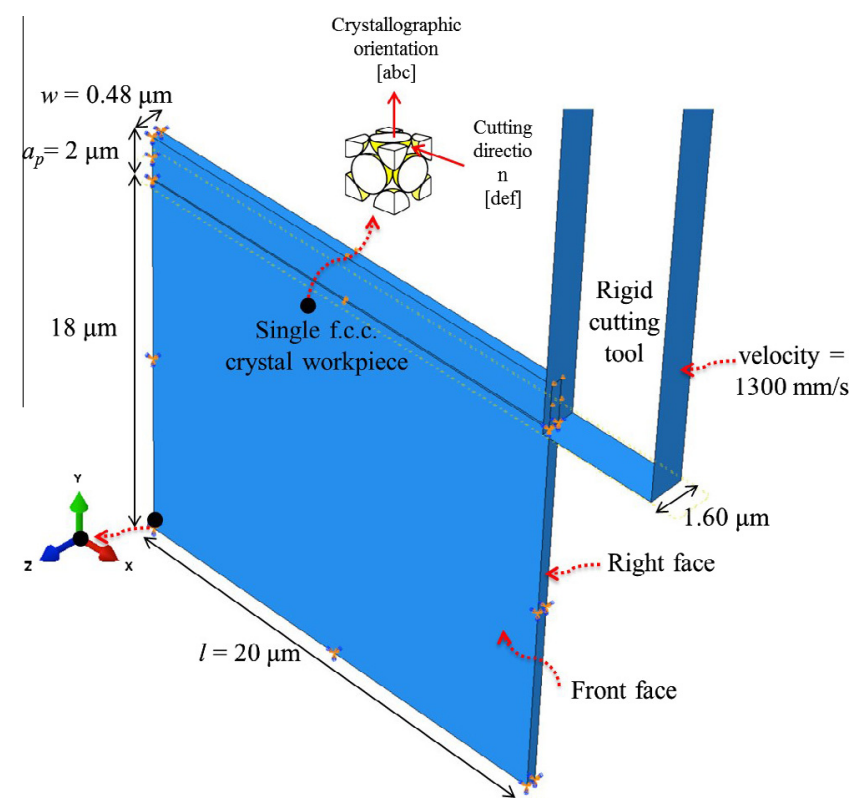

Fig. 1. Dimensions and orientations for orthogonal machining of single-crystal workpiece material (width of the tool, the arrow of the cutting).

The mesh of the workpiece with the advancing tool is shown in Fig. 2. A narrow band of elements with a height of $0.07 \mu \mathrm{m}$ suffers maximal damage during machining, which was deleted with increasing accumulated strain.

Three material parameters, $C_{11}=168.0 \mathrm{GPa}, C_{12}=121.4 \mathrm{GPa}$, $C_{44}=75.4 \mathrm{GPa}$, were used to define the elasticity tensor for a f.c.c. crystal [27]. Plasticity parameters used in the simulations are listed in Table 2.

\section{Results and discussion}

In this section, the results of FE simulations for orthogonal micro-machining of single crystal of $\mathrm{Cu}$ are presented. To study the influence of cutting direction and crystallographic orientation on the cutting force, two cutting directions, viz. $0^{\circ}$ and $90^{\circ}$, on three crystal planes, viz. (110), (111), (100) were chosen. The corresponding values are listed in Table 3 . The simulations were
Table 2

Plastic parameters used in FE simulations for copper single-crystal $[21,27,28]$.

\begin{tabular}{llllll}
\hline$\dot{\gamma}_{0}^{\alpha}\left(\mathrm{s}^{-1}\right)$ & $n$ & $q$ & $h_{0}(\mathrm{MPa})$ & $\left.g_{T}^{\alpha}\right|_{\mathrm{sat}}(\mathrm{MPa})$ & $\alpha_{T}$ \\
\hline $10^{-3}$ & 20 & 1 & 180 & 240 & 0.7 \\
$K(\mathrm{MPa} \mathrm{mm})$ & $\mu_{s}(\mathrm{GPa})$ & $b(\mathrm{~mm})$ & $\left.\rho_{S}\right|_{t=0}\left(\mathrm{~mm}^{-2}\right)$ & $\left.\rho\right|_{t=0}\left(\mathrm{~mm}^{-2}\right)$ & $\bar{S} / \bar{V}$ \\
0.04 & 42.0 & $2.55 \times 10^{-7}$ & $9 \times 10^{6}$ & $10^{6}$ & 4.36 \\
\hline
\end{tabular}

Table 3

Cutting direction setup ([def]) for different crystallographic orientations ([abc]) (see Fig. 1).

\begin{tabular}{lllll} 
Crystal orientation [abc] & & {$[110]$} & {$[111]$} & {$[100]$} \\
Cutting directions [def] & $0^{\circ}$ & {$[1-10]$} & {$[-110]$} & {$[011]$} \\
& $90^{\circ}$ & {$[001]$} & {$[11-2]$} & {$[01-1]$} \\
\hline
\end{tabular}

performed using different types of strain gradient crystal plasticity theories and conventional crystal plasticity theory.

\subsection{Effect of crystal orientation and cutting direction}

Evolution of the calculated cutting forces with an increasing cutting length for the combination of three cutting planes and two cutting directions is shown in Fig. 3. The cutting force was found to vary for different crystallographic orientations and cutting directions. For instance, while cutting on the (110) plane, the measured value of the cutting force at the [1-10] direction is larger compared to that in the [001] direction. The variation is less pronounced when cutting in the (111) plane. On the other hand, when cutting on the $(100)$ plane, the cutting forces were found to be identical for the cuttings at $0^{\circ}$ and $90^{\circ}$. This is due to the increased crystallographic symmetry of (100) plane in comparison to the other planes [11]. These findings are in good agreement qualitatively with the experiments performed by [10] for cutting of single-crystal copper. In this study, the obtained values are not compared quantitatively with those in [10] due to differences in the cutting conditions, which include tool geometry and contact conditions between tool and workpiece.

Fig. 3(d)-(f) demonstrate the obtained chip morphologies for different cutting planes and cutting directions of single-crystal copper. The chip shape was observed to be heavily influenced by crystallographic orientations and the cutting direction for cutting performed on the (110) and (111) planes. For cutting on the (100) plane the chip shape was found to be independent of the

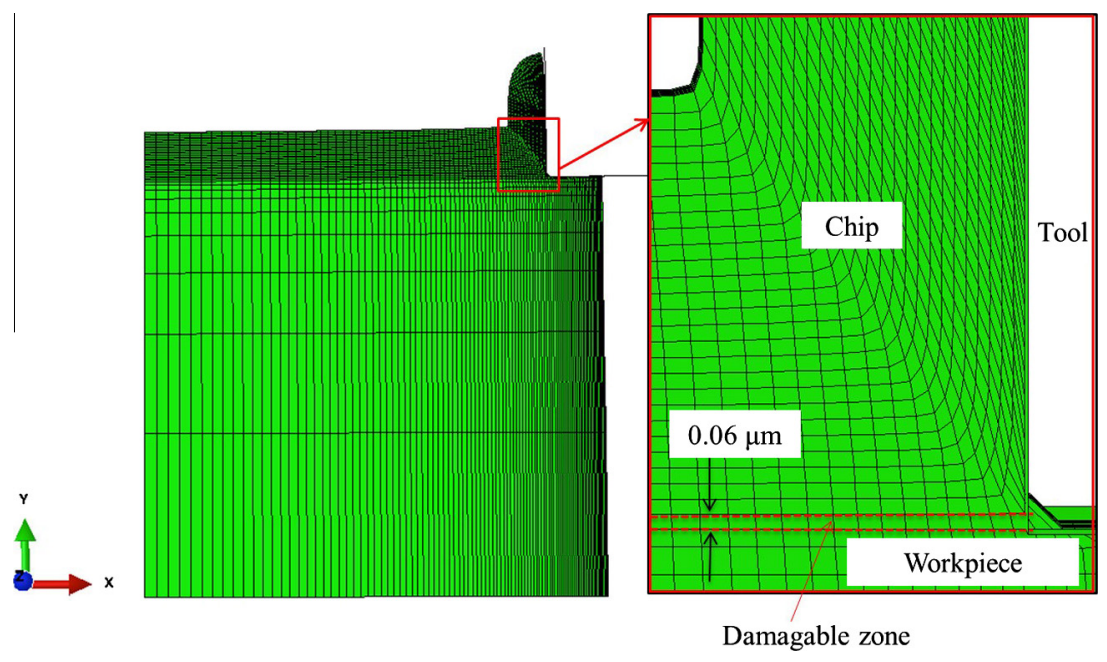

Fig. 2. 3D FE model of micro-machining with details of element-deletion module. 
(110)

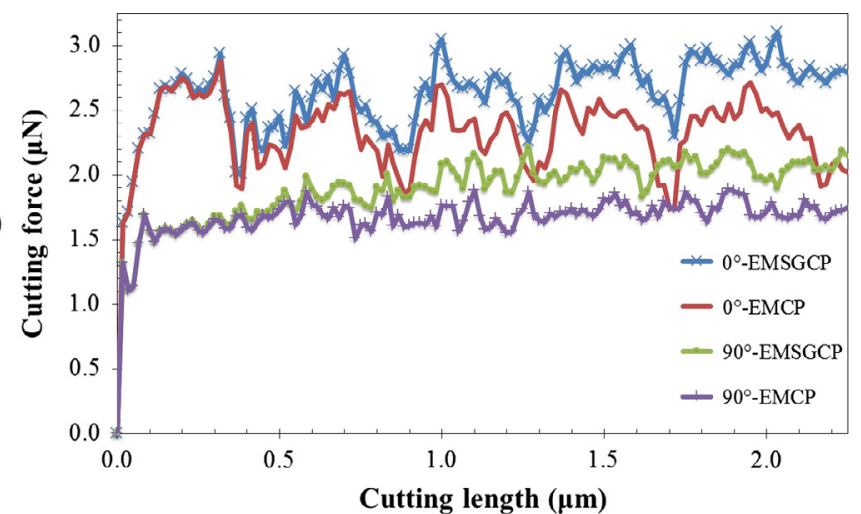

(a)

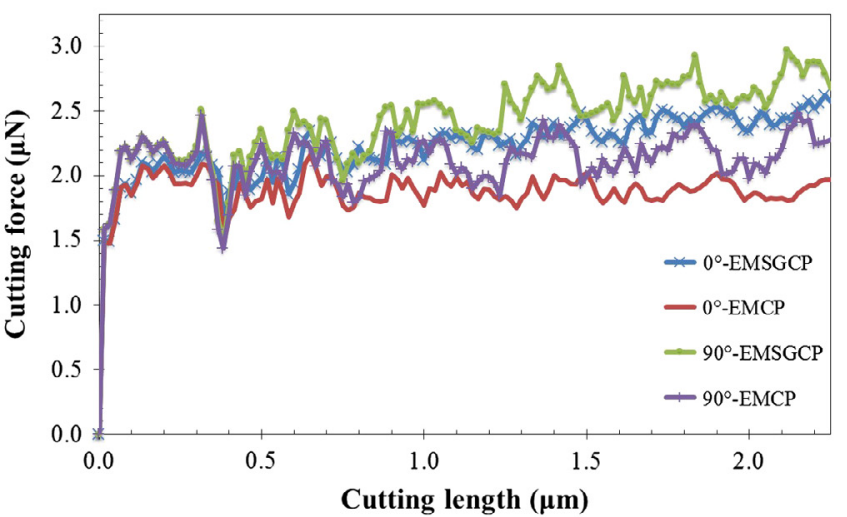

(b)

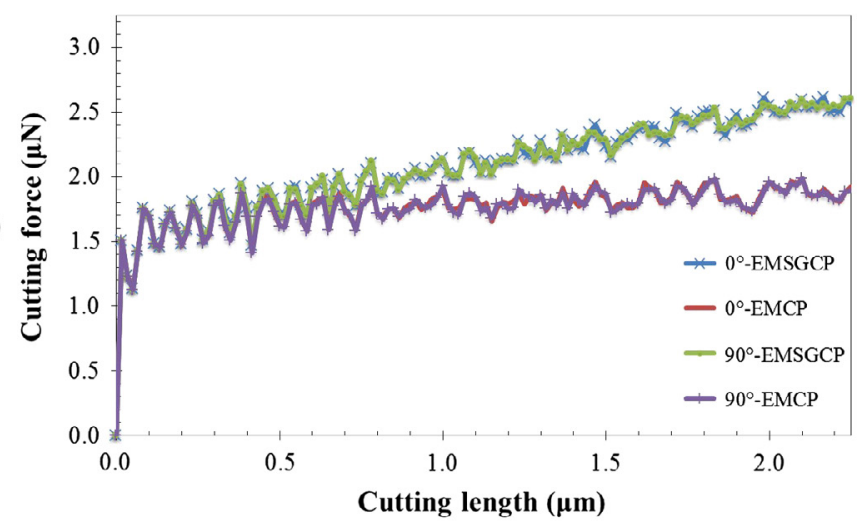

(c)
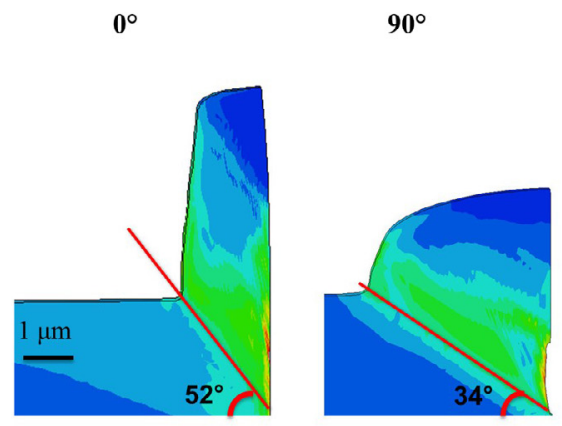

(d)
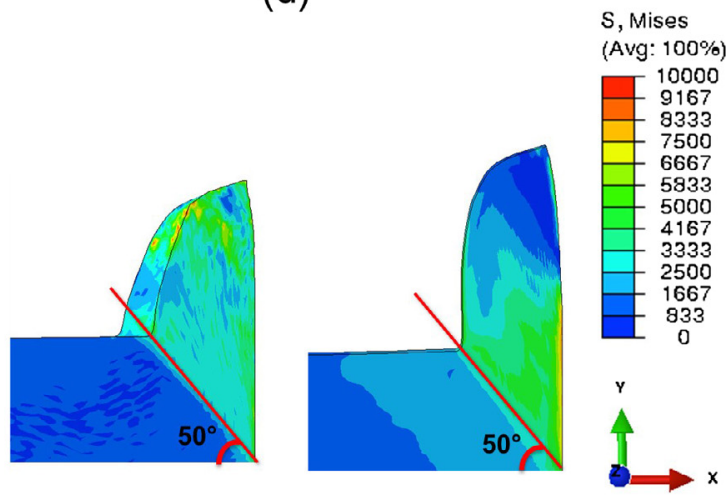

(e)
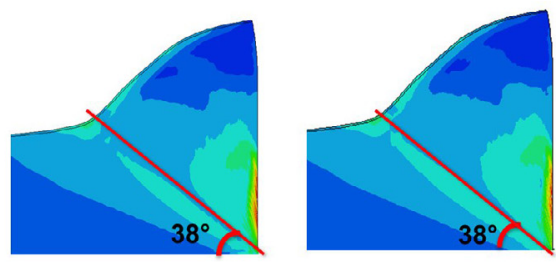

(f)

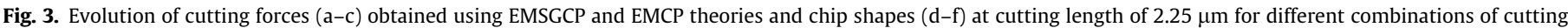
planes and cutting directions.

cutting direction, which manifests in the identical cutting-force responses (Fig. 3(c)).

To characterise the contribution of strain gradients and its evolution during the inhomogeneous cutting process, numerical experiments were carried out using the EMSGCP theory, which was then compared against EMCP (Fig. 3(a)-(c)). The results demonstrate that EMSGCP theory predicts a cutting force that increases with an increase in the cutting length; in contrast to the EMCP theory. These observations are valid irrespective of the crystal orientation and cutting directions. The difference in the predicted cutting force for the two theories was due to the fact that in the EMSGCP theory the strain-hardening rate was higher due to accounting for the evolution of strain gradients during deformation. The specific cutting energy, defined as the average of force magnitudes for the cutting lengths of $0.25 \mu \mathrm{m}$ and $2.25 \mu \mathrm{m}$, is normalized by the depth of cut $a_{p}$ and the workpiece's width $w$ (the resulting parameter is $F /\left(a_{p} w\right)$ ); it is listed in Table 4 as $\underline{F}_{\mathrm{EMSGCP}}$ and $\underline{F}_{\mathrm{EMCP}}$ for EMSGCP and EMCP, respectively. As expected, this value predicted with the EMSGCP theory was noticeably larger (12-17\%) when compared to the predictions based on the EMCP theory. It should be noted that the difference between the average energy magnitudes for the EMSGCP and EMCP theories becomes even larger when the cutting-energy values at larger cutting lengths are considered in calculating the average energy values. This difference (Table 4) demonstrates that the influence of strain gradients was sensitive to the crystallographic orientations of the single crystal 
Table 4

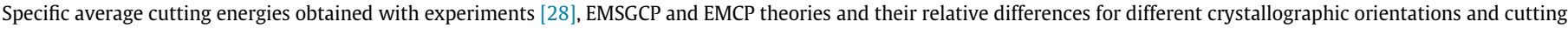
planes.

\begin{tabular}{|c|c|c|c|c|c|}
\hline Orientation & Cutting direction & $\underline{F}_{\text {EXP }}(\mathrm{MPa})$ & $\underline{F}_{\text {EMSGCP }}(\mathrm{MPa})$ & $\underline{F}_{\mathrm{EMCP}}(\mathrm{MPa})$ & $\left(\underline{F}_{\mathrm{EMSGCP}}-\underline{F}_{\mathrm{EMCP}}\right) / \underline{F}_{\mathrm{EMSGCP}}(\%)$ \\
\hline \multirow[t]{2}{*}[110]{} & $0^{\circ}$ & 2300 & 2777 & 2430 & 12.50 \\
\hline & $90^{\circ}$ & & 2035 & 1772 & 12.92 \\
\hline \multirow[t]{2}{*}[111]{} & $0^{\circ}$ & & 2371 & 1966 & 17.07 \\
\hline & $90^{\circ}$ & & 2571 & 2220 & 13.68 \\
\hline \multirow[t]{2}{*}[100]{} & $0^{\circ}$ & & 2253 & 1872 & 16.83 \\
\hline & $90^{\circ}$ & & 2253 & 1872 & 16.83 \\
\hline Average value & & 2300 & 2376 & 2022 & 14.89 \\
\hline
\end{tabular}

being cut and to the cutting direction; especially when cutting on the (111) plane. The obtained results were compared with the experimental data in [28] (unfortunately, orientation of the copper-crystal sample investigated in this experiment was not mentioned). This comparison demonstrates that for all the studied crystal orientations and cutting directions the average cutting energy $F_{\text {EMSGCP }}$ was in good agreement with the experimentally obtained value of specific cutting energy ( $\left.\underline{F}_{\text {Exp. }}\right)$ (Table 4$)$. Consequently, the predictions based on the EMCP theory was found to be lacking in accuracy.

\subsection{Effect of workpiece size in machining}

In this section, the influence of workpiece size on the machining response is studied. The modelled workpiece consisted of a single crystal of copper oriented in the (111) plane being cut in the $90^{\circ}$ direction. In addition to the workpiece with dimensions introduced in Section 2.2 (henceforth known as model A), an additional FE model of a workpiece with dimensions of $40 \mu \mathrm{m} \times 40 \mu \mathrm{m} \times$ $0.96 \mu \mathrm{m}$ (known as model B) was developed. The plastic parameters for each model are presented in Table 5. The finite-element size and machining parameters are identical in both models.

The specific average cutting-energy values for models A and B, obtained using different theoretical assumptions, are presented in Fig. 4. It is observed that the $F /\left(a_{p} . w\right)$ value for model $\mathrm{A}$ is higher compared to that for model $\mathrm{B}$ when predictions are based on the EMSGCP theory. The difference in specific cutting energies is less pronounced when predictions are based on the EMCP theory as the evolving strain gradients are not accounted for in this theory. On the other hand, the MBSGCP theory predicts a limited size effect in the simulations due to the fact that it accounts for the evolving strain gradients, with their magnitudes inversely proportional to the width of the workpiece material. Finally, the CP theory, as expected, does not predict any size effect as it does not account for any kind of GNDs or their evolution in its constitutive description.

Our study indicates that the EMSGCP theory is capable of capturing the classic 'size-effect' in machining problems which is otherwise widely reported in micromechanics. In traditional machining studies, an increase in a specific cutting force is typically reported when the cutting depth becomes comparable to the tool nose radius (i.e. transition to ploughing). This is typically attributed to the effect of geometry and friction at smaller cutting depths [29]. The modelling approach, as discussed here, elucidates the physical basis for higher cutting forces at smaller cutting depths. There is a need for further in-depth experimental studies

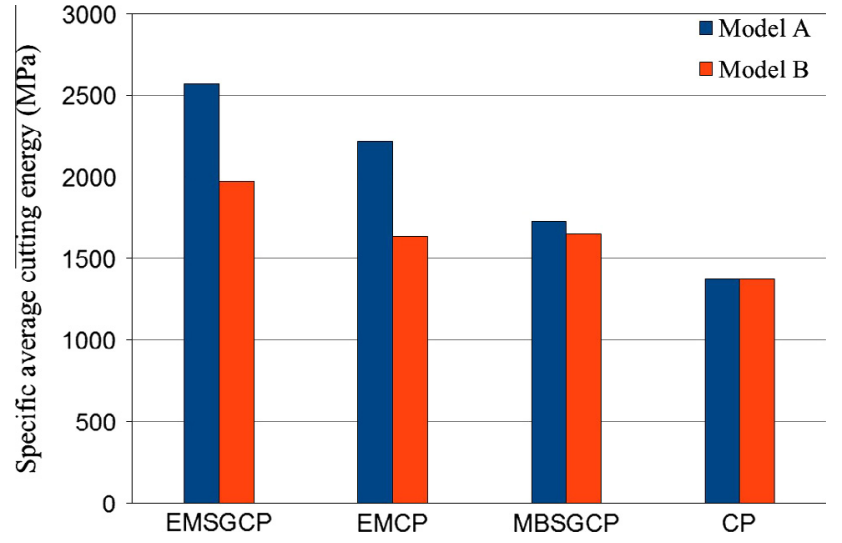

Fig. 4. Specific average cutting-energy values obtained for models A and B of singlecrystal (111) copper cut with direction of $90^{\circ}$ obtained using different theories.

of micromachining of single-crystal samples with different sizes. Once this is accomplished, validation/calibration tests will be implemented with the model.

\section{Concluding remarks}

In this paper, the influence of strain gradients and their evolution in the micro-machining of f.c.c. single-crystal copper for different crystallographic orientations and cutting directions was investigated using various flavours of strain-gradient theories and conventional crystal-plasticity theory. Clearly, there is a need for further experimental studies, which can then be used to validate our numerical predictions.

From our study, the following observations were made:

1. Strain gradients developing in the micro-machining process influence evolution of the cutting-force magnitude, more significantly at larger cutting lengths. This is sensitive to the crystallographic orientation of the single crystal being cut and also dependent on the cutting direction, especially for cutting on the (111) plane.

2. For cutting in the $(110)$ crystal orientation, the $90^{\circ}$ cutting direction is preferable to $0^{\circ}$ due to a lower cutting force imposed on the cutting tool in the former direction, which, in turn, is expected to yield better surface finish and a longer tool life. At the same time, for the (111) and (100) orientations both cutting directions lead to similar force levels.

Table 5

Material parameters for models A and B.

\begin{tabular}{|c|c|c|c|c|c|c|}
\hline Model & Dimension $(l \times h \times w)(\mu \mathrm{m})$ & $\bar{S} / \bar{V}$ & $\left.g_{S}^{\alpha}\right|_{t=0}(\mathrm{MPa})$ & $\left.g_{G}^{\alpha}\right|_{t=0}(\mathrm{MPa})$ & $\left.g_{T}^{\alpha}\right|_{t=0}(\mathrm{MPa})$ & $\left.g_{T}^{\alpha}\right|_{\text {sat }}(\mathrm{MPa})$ \\
\hline A & $20 \times 20 \times 0.48$ & 4.36 & 120 & 174.66 & 211.91 & 239.91 \\
\hline B & $40 \times 40 \times 0.96$ & 2.18 & 120 & 87.33 & 148.41 & 176.41 \\
\hline
\end{tabular}


3. The dimensions of the workpiece at micro-scale play an important role in its deformation response to the machining process. It was observed that smaller workpiece samples were harder to cut when compared to larger samples.

The developed scheme will be extended to machining of polycrystalline materials, accounting for different orientations of neighbouring grains in the workpiece. One important challenge on this way is a development of an adequate model of the grain boundary, which - as experiments demonstrate - affect the cutting process, especially at low depths of cut.

\section{Acknowledgement}

MD and TES acknowledge the KAUST baseline fund.

\section{References}

[1] J.R. Greer, J.T.M. De Hosson, Prog. Mater. Sci. 56 (2011) 654-724.

[2] T. Schaller, L. Bohn, J. Mayer, K. Schubert, Prec. Eng. 23 (1999) 229-235.

[3] H. Suzuki, T. Moriwaki, Y. Yamamoto, Y. Goto, CIRP Annals-Manuf. Technol. 56 (2007) 131-134

[4] E. Brinksmeier, O. Riemer, A. Gessenharter, L. Autschbach, CIRP Annals-Manuf. Technol. 53 (2004) 247-250.

[5] X. Cheng, K. Nakamoto, M. Sugai, S. Matsumoto, Z.G. Wang, K. Yamazaki, CIRP Annals-Manuf. Technol. 57 (2008) 415-420.
[6] T. Moriwaki, CIRP Annals-Manuf. Technol. 38 (1989) 115-118.

[7] B.F. Von Turkovich, J. Temple Black, J. Eng. Industry 92 (1970) 130.

[8] W.B. Lee, S. To, C.F. Cheung, Scr. Mater. 42 (2000) 937-945.

[9] M. Sato, Y. Kato, K. Tsutiya, Trans. Japan Inst. Metals 20 (8) (1979).

[10] M. Zhou, B.K.A. Ngoi, Proc. Inst. Mech. Eng., Part B: J. Eng. Manuf. 215 (2001) 13-19.

[11] N. Kota, O.B. Ozdoganlar, Mach. Sci. Technol. 14 (2010) 102-127.

[12] E. Demir, J. Mach. Tools Manuf. 48 (2008) 1592-1598.

[13] H.M. Pen, Y.C. Liang, X.C. Luo, Q.S. Bai, S. Goel, J.M. Ritchie, Comp. Mater. Sci. 50 (2011) 3431-3441.

[14] R. Komanduri, N. Chandrasekaran, L.M. Raff, Wear 242 (2000) 60-88.

[15] S.A. Zahedi, M. Demiral, A. Roy, V.V. Silberschmidt, Comp. Mater. Sci. 78 (2013) 104-109.

[16] S.A. Tajalli, M.R. Movahhedy, J. Akbari, Comp. Mater. Sci. 86 (2014) 79-87.

[17] K. McElhaney, J. Vlassak, W. Nix, J. Mater. Res. 13 (1998) 1300-1306.

[18] N.A. Fleck, G.M. Muller, M.F. Ashby, J.W. Hutchinson, Acta Metallur. Mater. 42 (1994) 475-487.

[19] J. Stölken, A. Evans, Acta Mater. 46 (1998) 5109-5115.

[20] N. Stelmashenko, M. Walls, L. Brown, Y.V. Milman, Acta Metallur. Mater. 41 (1993) 2855-2865.

[21] M. Demiral, Enhanced gradient crystal-plasticity study of size effects in b.c.c. metal, PhD thesis, Loughborough University, UK, 2012

[22] M. Demiral, A. Roy, V.V. Silberschmidt, Comp. Mater. Sci. 79 (2013) 896-902.

[23] D. Peirce, R. Asaro, A. Needleman, Acta Metallur. 30 (1982) 1087-1119.

[24] D. Systemes, Abaqus 6. 11 Analysis User Manual (2011).

[25] C. Han, H. Gao, Y. Huang, W.D. Nix, J. Mech. Phys. Solids 53 (2005) 1188-1203.

[26] A. Shrot, M. Bäker, Comp. Mater. Sci. 52 (2012) 298-304.

[27] Y. Wang, D. Raabe, C. Klüber, F. Roters, Acta Mater. 52 (2004) 2229-2238.

[28] Y. Furukawa, N. Moronuki, Annals CIRP. 37 (1988) 113-116.

[29] C.-F. Wyen, K. Wegener, CIRP Annals-Manuf. Technol. 59 (2010) 93-96. 